\title{
Switchable dual-wavelength mode-locked fiber laser source for in-PCF parametric frequency conversion applied to CARS microscopy
}

\author{
I. Aporta, M. A. Quintela, J. M. López-Higuera
}

\begin{abstract}
A self-started, switchable dual-wavelength allpolarization-maintaining (PM) mode-locked (ML) fiber laser for optical parametric generation (OPG) in photonic crystal fiber (PCF) applied to CARS (Coherent anti-Stokes Raman scattering) microscopy is reported in this article. The complete laser setup is built in an all-fiber structure composed of commercial elements. It is based on a linear cavity with an active medium, namely Ytterbium doped fiber (YDF), a pair of fiber Bragg gratings (FBG) as switchable partial mirrors between $1031.5 \mathrm{~nm}$ and $1049.7 \mathrm{~nm}$ and a semiconductor saturable absorber mirror (SESAM) functioning also as the mode-locking device. The seed laser is amplified to $650 \mathrm{~mW}$ average power by two Yb dopedfiber amplifiers (YDFA). The source delivers a pulse-train output of 30.9 ps at 1031.5 ps and 31.6 ps at 1049.7, a 3.1 MHz repetition rate, and over $200 \mathrm{~nJ}$ pulse energy and $6 \mathrm{~kW}$ of peak power at fundamental mode-locked operation. The properties of this switchable dual-wavelength source ensures the correct OPG by degenerated four-wave mixing (DFWM) in PCF fulfilling the phase matching condition which corresponds to CARS resonance of $2850 \mathrm{~cm}^{-1}$ (CH3-O molecular bonds) at $1031.5 \mathrm{~nm}$ and 1475 $\mathrm{cm}^{-1}$ (CH2 $\delta$ molecular bonds) at $1049.7 \mathrm{~nm}$.
\end{abstract}

Index Terms-Optical fiber lasers, Mode locked lasers, Laser tuning, Fiber nonlinear optics, Fiber Bragg gratings.

\section{INTRODUCTION}

$\mathrm{O}$ VER the past few years, switchable and wavelengthtunable passively mode-locked fiber lasers have attracted a lot of interest because of their versatile applications in fiber sensing, materials processing and micromachining, as well as optical signal communications, spectroscopy and biomedical applications [1-8]. These kind of fiber lasers have been developed and experimentally demonstrated offering many advantages with respect to solid-state lasers because of their high environmental stability, low cost, good beam quality, compact design, robustness and heat dissipation management [1, 9-13]. Generally, passive mode-locking fiber

Manuscript received. This work was supported by the Spanish Comisión Interministerial de Ciencia y Tecnología within project TEC2016-76021-C2 $\mathrm{R}$ and Feder funds and the Spanish Ministry of Education and Culture, within the scholarship FPU14/02196.

I. Aporta is with the Photonics Engineering Group and CIBER-bbn, Universidad de Cantabria, 39005 Santander, Spain (e-mail: aportai@unican.es)

M. A. Quintela and J. M. Lopez-Higuera are with the Photonics Engineering Group, CIBER-bbn and IDIVAL, Universidad de Cantabria, 39005 Santander, Spain (e-mail: angeles.quintela@unican.es; miguel.lopezhiguera@unican.es) lasers have been achieved via different types of techniques including nonlinear polarization rotation (NPR), nonlinear optical loop mirror (NOLM), graphene layering, carbon nanotube saturable absorbers (CNT) and semiconductor saturable absorber mirrors (SESAM) [14-21]. Among all the saturable absorbers (SAs), SESAMs have become an ideal option because of their technological maturity and commercial availability [22]. Furthermore, the development of SESAM devices has expanded the applicability in the industry of lasers due to its reliable design and flexibility [23], allowing easyhandle self-starting passive mode-locking by properly choosing the cavity design and controlling the growth parameters of the SESAM semiconductor material [24]. In particular, the advances in ytterbium-doped ultrafast fiber lasers with SESAM technology have demonstrated that $\mathrm{Yb}$ doped fibers are the best option as the gain medium for generation and amplification of SESAM-based mode-locking ultrashort pulses around $1 \mu \mathrm{m}$ wavelength range $[25,26]$ as a consequence of its broad absorption and emission bandwidth, large saturation fluences and high optical conversion efficiencies [27-31].

All-fiber passive mode-locking systems are a popular pulse formation mechanism to achieve switchable and tunable wavelength lasers due to their low losses and cost-efficiency [32]. This type of fiber laser has been widely investigated with the aforementioned methods, i.e. NPR [33], NOLM [34], CNT [35] and SESAM [36]. However, the NPR-based and NOLMbased mode locked fiber lasers are polarization-sensitive as well as intensity-dependent, which implies a complicated adjustment in an all-fiber structure [37, 38]. SESAM technology has been used in many tunable-wavelength pulsed fiber lasers [24, 39] due to the inherent benefits of polarization insensitivity, being the main commercial option as the ML device in this kind of sources. Additionally, FBGs are key elements in wavelength selection through spectral filtering effects, and play an important role [40] in the achievement of tunable and switchable wavelength ML fiber lasers due to their capability of being tunable by applying strain [41] and temperature variations [42] and additional advantages such as ease of handling, low losses, free-alignment and fiber compatibility [43, 44]. Furthermore, the inclusion of FBGs in the all-fiber laser structure stabilizes mode-locking and limits pulse duration to the picosecond range in the fundamental ML operation because of its relatively narrow reflection bandwidth. This is beneficial for CARS microscopy applications because pulse duration control in the tens-ofpicoseconds range allows for a good compromise between the 
required high peak power and the adequate spectral resolution $[8,45]$. Moreover, it has been probed that DFWM in commercial endlessly single-mode PCF allows for high conversion efficiencies, generating new frequencies with high spectral densities [46] and ensuring the necessary mode overlap of the generated signals even if they are spectrally widely separated. Hence, the temporal overlapping needs to be controlled to ensure a high resolution without experimental difficulties, since the use of sub-ps or 1-2 ps pulses only results in moderate resolution [47] requiring delay lines to control any lack of temporal overlapping. In addition, the achievement of pulse trains in the tens-of-picoseconds range reduces spectral broadening due to self-phase modulation (SPM) of the driving pulses, allowing narrow bandwidth signal generation in the nonlinear frequency conversion process.

In the present paper, a new experimental implementation of a compact all-fiber, free-alignment, self-started picosecond switchable dual-wavelength ML laser source has been demonstrated. The complete setup is built out of PM commercial fiber components making a cost-efficient and environmentally-stable structure. The picosecond master oscillator delivers a train of pulses with low $\mathrm{MHz}$ repetition rate and is based on $\mathrm{Yb}$-doped fiber, two FBGs connected in parallel as the switchable wavelength device in the extreme of the cavity and a fiber-coupled SESAM as the ML device (saturable absorber mirror). The compactness of the system mainly relies in the construction of the Yb-doped fiber amplifiers with PM single-mode fiber components, due to the reduced average power requirements caused by the low $\mathrm{MHz}$ repetition rate and picosecond pulse duration. The experimental results show a switchable dual-wavelength output laser between $1031.5 \mathrm{~nm}$ and $1049.7 \mathrm{~nm}$, with $0.72 \mathrm{~nm}$ and $0.69 \mathrm{~nm}$ of 3-dB bandwidth respectively owing to the SPM spectral broadening. The output pulses are $30.9 \mathrm{ps}$ at $1031.5 \mathrm{ps}$ and $31.6 \mathrm{ps}$ at 1049.7 , with a $3.1 \mathrm{MHz}$ repetition rate, over $200 \mathrm{~nJ}$ pulse energy and $6 \mathrm{~kW}$ peak power. This switchable dual-wavelength laser structure is well suited to drive the FWM in PCF generating the signals at a frequency difference matching the CARS resonance around $2850 \mathrm{~cm}^{-1}$ (CH3-O molecular bonds) and around $1475 \mathrm{~cm}^{-1}(\mathrm{CH} 2 \delta$ molecular bonds).

\section{EXPERIMENTAL SETUP AND PRINCIPLE}

The configuration of the proposed switchable dualwavelength ML fiber laser is shown schematically in Fig. 1. The system is based on a passively ML oscillator, which is amplified by a two-stage YDF amplifier. The design of the master oscillator is a linear cavity composed of two FBGs in parallel connection through a 50:50 optical coupler, a 1 meterlong Yb-doped fiber (Nufern PM-YSF-LO) as the gain medium, a delay-line of 25 meters of PM fiber (Nufern 980XP), an in-line polarizer and a SESAM (Batop GmbH) which is butt-coupled and has an absorptance of $40 \%$, a modulation depth of $29 \%$, a saturation fluence of $90 \mu \mathrm{J} / \mathrm{cm}^{2}$, a non-saturable loss of $11 \%$ and a relaxation time of $9 \mathrm{ps}$. The FBGs are placed on a Peltier and have a center wavelength at $1031.5 \mathrm{~nm}$ and $1049.7 \mathrm{~nm}$, a bandwidth of $0.31 \mathrm{~nm}$ and 0.29 $\mathrm{nm}$, and a reflectivity of $89 \%$ and $92 \%$ respectively. The fibers

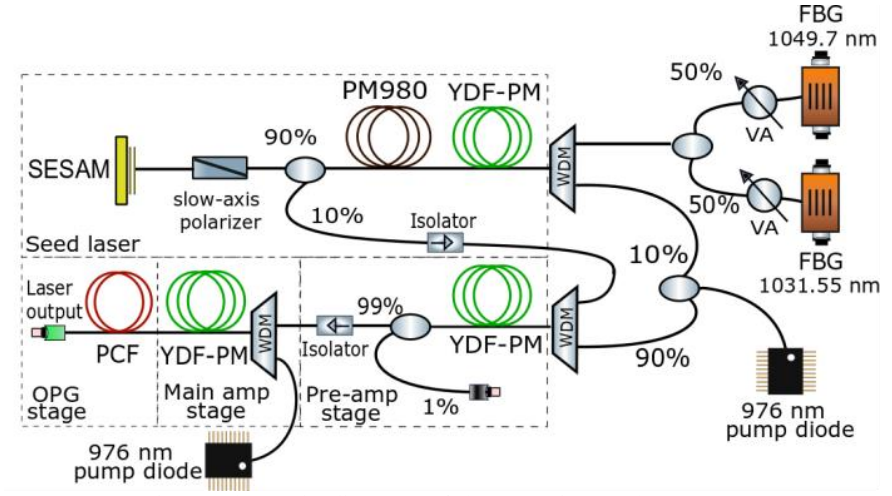

Fig.1 Schematic diagram of the experimental setup.

between the 50:50 coupler and the FBGs has been looped around two tubular mounts functioning as variable optical attenuators (VOA) by fixing single mode fibers to micrometric screws [48]. This simple method allows for an adjustment of the losses changing the curvature radius of the fibers, while avoiding insertion losses [49]. A 90:10 optical coupler has been used to provide a laser output through the $10 \%$ port while the $90 \%$ port is engaged to feedback into the linear cavity. The Yd-doped fiber is pumped by a $976 \mathrm{~nm}$ laser diode (LD) of $750 \mathrm{~mW}$ optical power which is coupled to a 90:10 optical coupler delivering the $10 \%$ of the input power to the master oscillator via a 976/1030 wavelength division multiplexer (WDM). The oscillator output is connected to an isolator before the two stage fiber amplifier, to suppress possible back reflections protecting the oscillator and the ML operation. The first amplifier stage is based on a 1.2 meters-long Yb-doped fiber (Nufern PM-YSF-HI) and is pumped by the $90 \%$ of the LD input power which is engaged to the coupler as is depicted in Fig.1. A 99:1 fiber coupler is introduced in the stage to monitor and control the behavior of the source through the $1 \%$ port. The second amplifier stage is composed of a $40 \mathrm{~cm}-$ long double-clad Yb-doped fiber (Liekki Yb1200-6/125DC-PM) pumped through the core with the overall power given by a $750 \mathrm{~mW} 976 \mathrm{~nm}$ LD. Both stages are connected through a fiber isolator. The OPG based on DFWM is performed on a 1.6 meters-long PCF (LMA-5-PM NKT Photonics). An optical spectrum analyzer (Yokogawa AQ6373B) with a 0.02 $\mathrm{nm}$ resolution, an oscilloscope (Tektronix TBS1052B), an auto-correlator (Femtochrome FR-103XL) and a $1 \mathrm{GHz}$ photoreceiver (Thorlabs DET02AFC) have been used for spectral and temporal analysis of the source. An electric spectrum analyzer (R\&S FPS) and a power meter (ThorlabsPM100D) have been utilized to obtain the RF spectrum of the source and the laser output power.

The experimental setup depicted in Fig.1 allows ML-based pulse formation through forward core-pumping of the YDF in the master oscillator linear cavity. The pair of switchable FBGs determines the wavelength emission of the source and each one is selected adjusting in-fiber losses through custombuilt VOAs. The FBG is used to stabilize ML operation and to generate spectrally narrow pulses which is a must in order to match the requirements of the FWM process and, hence, CARS resonance. The in-line polarizer selects the slow-axis polarization suppressing the optical field polarized along the fast axis, ensuring an environmentally-stable operation regime against the interaction of polarized transversal modes. 


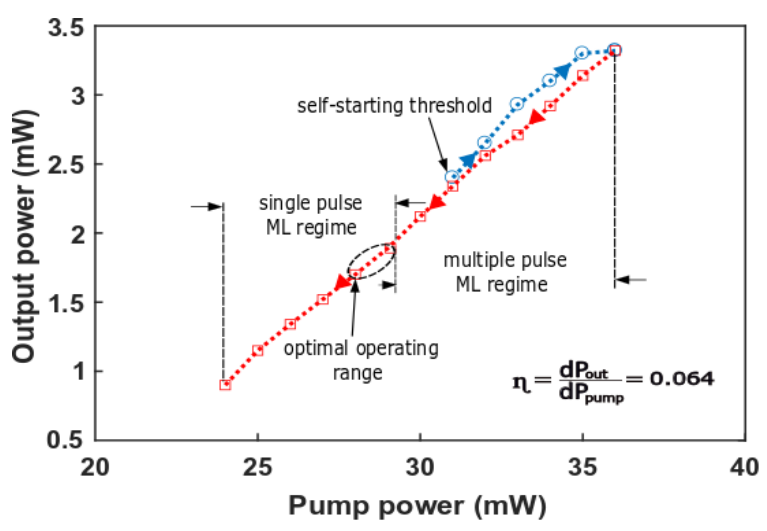

Fig.2 Hysteresis cycle of the ML operation regime at $1031.5 \mathrm{~nm}$ wavelength emission.

The self-started ML operation is easily obtained at lower pump power due to the cavity configuration and the election of the SESAM parameters. Moreover, the wavelength emission tunability of the ML laser source is achieved through the application of controlled temperature of the FBG with a Peltier device [50]. In this manner, the switchable-wavelength emission of the pulsed fiber laser is precisely adjusted by matching the required FWM conditions of CARS CHstretching vibration around $2850 \mathrm{~cm}^{-1}(\mathrm{CH} 3-\mathrm{O}$ molecular bonds) and $1475 \mathrm{~cm}^{-1}$ (CH2 $\delta$ molecular bonds), and maintaining the temporal characteristics despite any existing spectral shift. In addition, amplification of the pulsed laser oscillator through the two YDF amplification stages leads to an adequate average optical power, which in combination of a low $\mathrm{MHz}$ repetition rate and picosecond-range pulse duration, makes the system able to reach the necessary peak power to drive the mentioned FWM process in PCF.

\section{RESULTS AND DISCUSSION.}

In this experimental setup, the self-started ML operation is achieved when pump power exceeds the threshold of $31 \mathrm{~mW}$ and $32.8 \mathrm{~mW}$ at $1031.5 \mathrm{~nm}$ and $1049.7 \mathrm{~nm}$ wavelengths respectively. The use of a wide-spectral range SESAM with flattened reflection and the operation range of the utilized commercial wavelength-sensitive components allows for switchable-wavelength emission selection with a difference of $20 \mathrm{~nm}$ without considerable increment of the pump power. Fig. 2 shows the hysteresis cycle of the $1031.5 \mathrm{~nm}$ laser as a function of pump power. As it can be seen, the self-starting threshold initiates the ML operation in multiple-pulse regime. For this reason, once the ML state has been initiated, pump power has to be decreased to work in the single-pulse regime and thus, in the optimal operating range shown in Fig.2. The stable ML regime of the seed laser operating at $1031.5 \mathrm{~nm}$ delivers a pulse train of 32.2 to $37.5 \mathrm{ps}$, depending on the pump level. At a launched pump power of $28.5 \mathrm{~mW}$, the oscillator generates pulses with an average power of $1.85 \mathrm{~mW}$, a slope efficiency of $6.4 \%$, a duration of $32.2 \mathrm{ps,} \mathrm{a} \mathrm{spectral}$ width of $0.19 \mathrm{~nm}$, a pulse energy of $0.53 \mathrm{~nJ}$ and a repetition rate of $3.112 \mathrm{MHz}$ as is depicted in Fig. 3. Fig .4 shows the produced pulses at $1049.7 \mathrm{~nm}$ with a pump power of 30.2 $\mathrm{mW}$, yielding $33.1 \mathrm{ps}$ pulse duration, $0.17 \mathrm{~nm}$ spectral width, $0.49 \mathrm{~nJ}$ pulse energy, a repetition rate of $3.167 \mathrm{MHz}$ and an output power of $1.6 \mathrm{~mW}$. The negligible difference between both repetition rates lies on the variation of the fiber longitude of the FBGs and the coupler ports. The total cavity length is estimated over 33 meters long, with a 28 m delay line of PM980 fiber to fix the repetition rate.
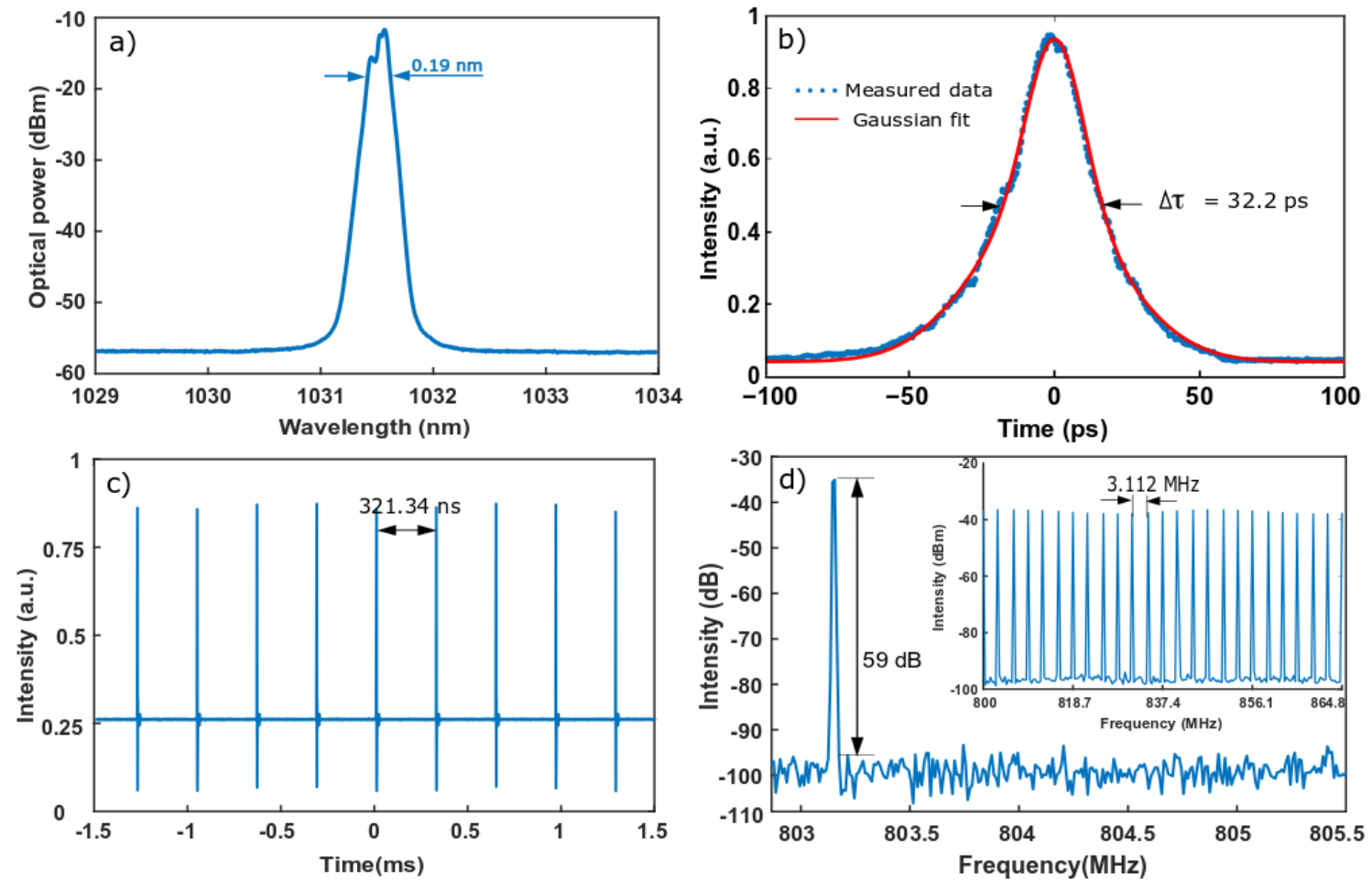

Fig.3 a) Optical spectrum, b) auto-correlation trace, c) temporal trace and d) RF spectrum of the $1031.5 \mathrm{~nm}$ ML fiber laser. 

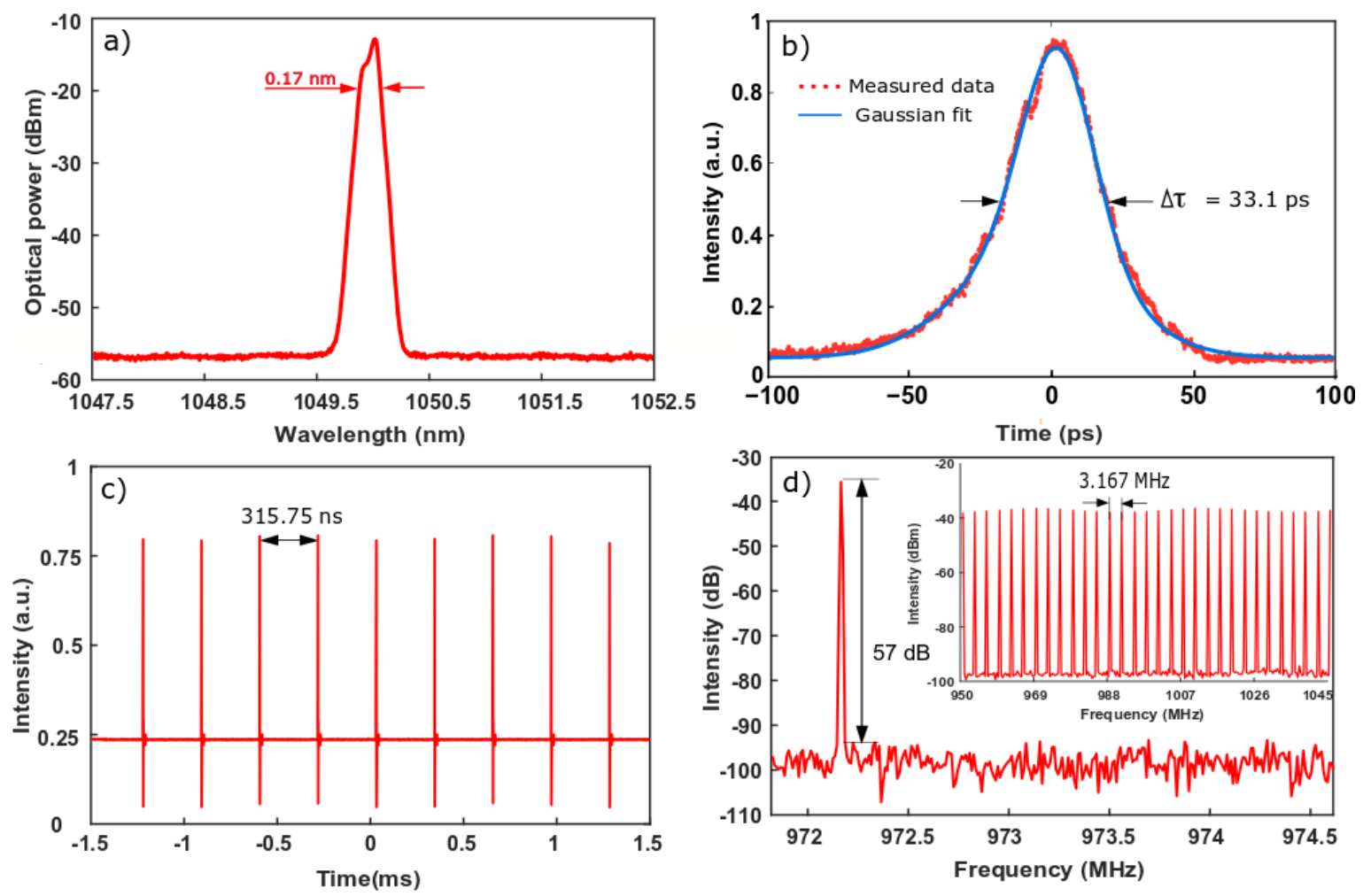

Fig.4 a) Optical spectrum, b) auto-correlation trace, c) temporal trace and d) RF spectrum of the $1049.7 \mathrm{~nm}$ ML fiber laser.

The signal-to-noise ratio (SNR) of the RF spectrum shown in Fig. 3d) and Fig. 4d) is nearly $60 \mathrm{~dB}$, which indicates a high mode-locking stability in both wavelengths laser emission. The stability study of the oscillator in both wavelength emissions shows a standard deviation below the $1.5 \%$.

The seed laser is launched into a two-stage YDF amplification configuration. The pre-amp stage uses $1.2 \mathrm{~m}$ of active fiber with a pump absorption of $250 \mathrm{~dB} / \mathrm{m}$ and is pumped with the $90 \%$ output port of the $976 \mathrm{~nm}$ LD used to generate the pulses in the oscillator. The total optical power pumped in the pre-amp is $515 \mathrm{~mW}$, delivering a seed with an output power of $\sim 60 \mathrm{~mW}$ (there are barely variations depending on the selected wavelength emission due to the gain profile of the YDF). The pre-amplified seed is launched into a $0.45 \mathrm{~m}$ main amplification fiber with a pump absorption of $1200 \mathrm{~dB} / \mathrm{m}$ which is pumped with the overall power of a 750 $\mathrm{mW} 976 \mathrm{~nm}$ LD. After the main amplification stage, a pulse train with an average power of $650 \mathrm{~mW}$ is obtained, resulting in a peak power of up to $6 \mathrm{~kW}$. This laser structure has a high slope efficiency of $\sim 43 \%$ which in turn implies a very good cost-efficiency ratio. The output spectra and the pulse width of the $1031.5 \mathrm{~nm}$ amplified seed laser in each amp-stage (preamplification and main amplification) is given in Fig 5. The temporal pulse width of the laser source remains almost constant (Fig.5b). Measured auto-correlation traces after the amplification stages yield a pulse duration of $30.9 \mathrm{ps}$ and 31.6 ps at $1031.5 \mathrm{~nm}$ and $1049.7 \mathrm{~nm}$ respectively, which barely differs from the ones measured at the oscillator output. However, pulses are spectrally broadened by nonlinear SPM effect due to the high peak power reached through the YDF amplifiers, showing significant SPM lateral structures depicted in Fig5a). The spectrum after the YDF amplifiers is broadened to 0.72 at $1031.5 \mathrm{~nm}$ and $0.69 \mathrm{~nm}$ at $1049.7 \mathrm{~nm}$.
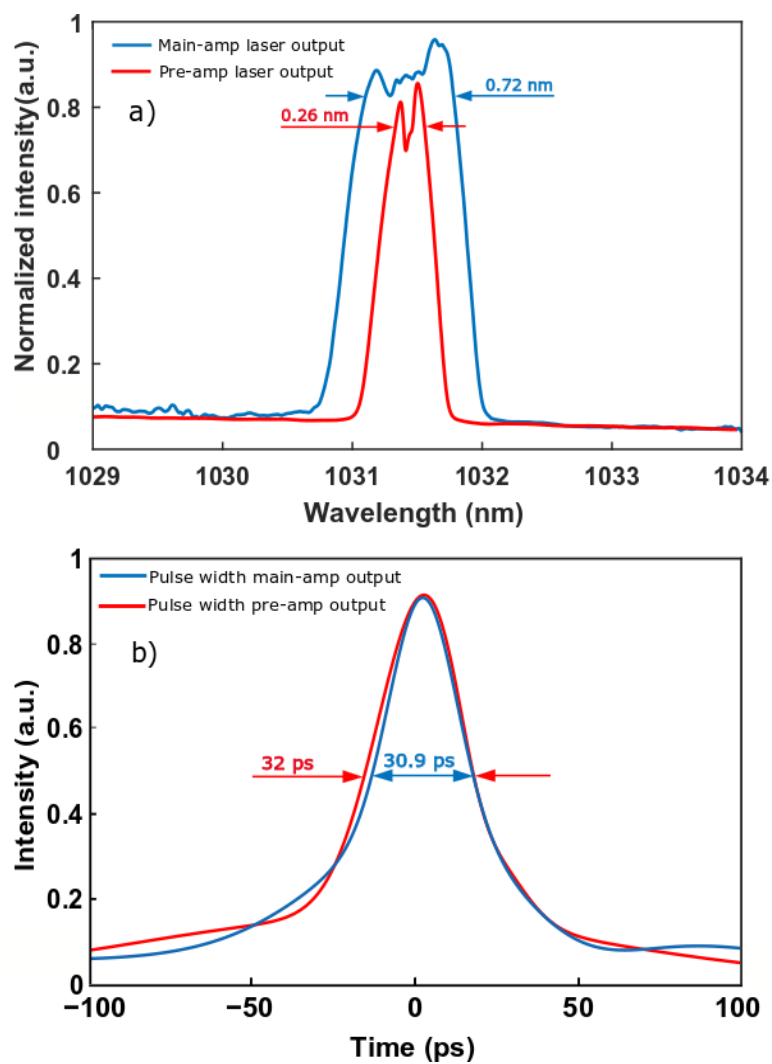

Fig.5 a) Optical spectrum and b) pulse duration of the $1031.5 \mathrm{~nm}$ amplified ML seed laser. 


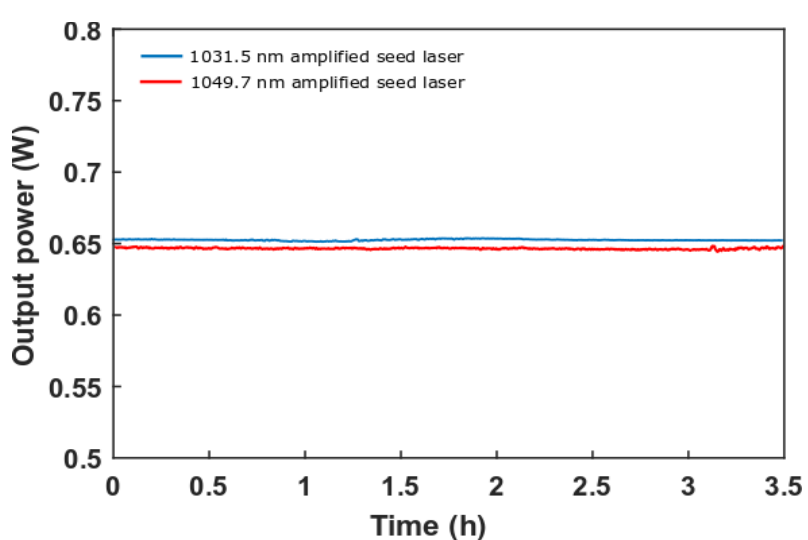

Fig.6. Output power variations of both wavelength laser emission.

Additionally, an average output power stability study of the laser has been illustrated in Fig.6. The study has been performed with a confidence level (CL) of $100 \%$ and over 20000 samples for each wavelength emission (3.5 hour-long measurements). In this stability studio, the overall standard deviation across wavelengths has been utilized to analyze output variations of the laser showing a value of $0.12 \%$ and $0.23 \%$ with an average power of $653 \mathrm{~mW}$ and $648 \mathrm{~mW}$ at $1031.5 \mathrm{~nm}$ and $1049.7 \mathrm{~nm}$ respectively. These experimental results grant very high stability with negligible power variations, which is ideal to perform OPG in PCF.

To drive this nonlinear process, simulations of PCFs based on FEM [51, 52] and numerical simulations of the FWM process based on [53] have been performed. As a result of these simulations, a commercially available PCF has been selected for this purpose. The fiber is a cost-effective endlessly single mode PCF developed by NKT Photonics named LMA-5 PM. This fiber has air holes in a hexagonal structure composed by 4 rings, with a pitch of $3.2 \mu \mathrm{m}$ and an air hole diameter of $1.5 \mu \mathrm{m}$ and is depicted in Fig. 7a). The mentioned PCF has the appropriate dispersion parameter to fulfill the requirements for FWM signal generation. Fig 7a) illustrates the FWM phase-matching diagram of signal generation in blue (anti-Stokes component) and the FWM frequency shift from respect to pump in red. Accordingly, the desired frequency difference between the FWM signal and the pump are obtained matching the CARS resonances of 2850 $\mathrm{cm}^{-1}$ (CH3-O molecular bonds) at $1031.5 \mathrm{~nm}$ and $1475 \mathrm{~cm}^{-1}$ $(\mathrm{CH} 2 \delta$ molecular bonds) at $1049.7 \mathrm{~nm}$ (calculated for a peak power of $6 \mathrm{~kW}$ and plotted in the proper figure).

In this experimental set-up, the fusion splice between the main YDF-based amplifier and the PCF is critical to obtain a good efficiency in the non-linear frequency conversion process. After previous alignment of the panda structure of both fibers, the optimized splice procedure keeps a very small collapse length of the PCF air holes and allows to obtain an experimental value of $73 \%$ coupling efficiency, which is an excellent ratio. The PCF output is $45^{\circ}$ angled-cut and placed on a bare fiber terminator with a FC / PC connector to perform the spectral measurements. The auto-correlation traces and the output power measurements of each FWM component have been achieved using optical filters after collimation of the laser output beam. As seen in Fig. 7b), the OPG based on DFWM in the PCF LMA-5-PM has been achieved showing the output spectra when the laser is emitting at both wavelengths. The experimental results show a power difference of $11 \mathrm{~dB}$ between the pump component and the anti-Stokes signal generated, which are the CARS signals used to match the desired resonance frequencies. In this case, the generated anti-Stokes spectra are centered at $794 \mathrm{~nm}$ (1031.5 $\mathrm{nm}$ pump wavelength) and $903 \mathrm{~nm}$ (1049.7 nm pump wavelength) matching the frequency separations of 2899 $\mathrm{cm}^{-1}$ and $1550 \mathrm{~cm}^{-1}$ respectively. The $3-\mathrm{dB}$ spectral width of the pump components has been broadened due to the high peak-power and the non-linear conversion effect to $1.3 \mathrm{~nm}$ and the anti-Stokes signal generated have a 3-dB spectral width of $4 \mathrm{~nm}$ at $794 \mathrm{mn}$ and $4.3 \mathrm{~nm}$ at $903 \mathrm{~nm}$. The measured autocorrelation traces of the pump $(1031.5 \mathrm{~nm})$ and anti-Stokes component $(794 \mathrm{~nm})$ after the frequency conversion in PCF are shown in Fig.9. The temporal measurements yield a pulse duration of $24.4 \mathrm{ps}$ at $794 \mathrm{~nm}$ and $23.1 \mathrm{ps}$ at $903 \mathrm{~nm}$. In the worst case, the pulse duration of the signals ensures a good overlap for the CARS process, considering the temporary walk-off between pump and anti-Stokes signal in the PCF due to the mismatch caused by group-velocity (less than 0.1 $\mathrm{ps} / \mathrm{cm})$. The power measurements at the end of the laser source output shows over $40 \mathrm{~mW}$ of FWM anti-Stokes signal at both pump wavelength. Owing to the needs of CARS imaging, the power level of tens of $\mathrm{mW}$ of the generated signals are more than enough for this application. Based on the experimental results, this alignment-free configuration ensures a very good temporal and spectral response of the CARS signals and high efficiency conversion, fulfilling the purpose of the laser source and making suitable to be applied on CARS microscopy as shown in [54].
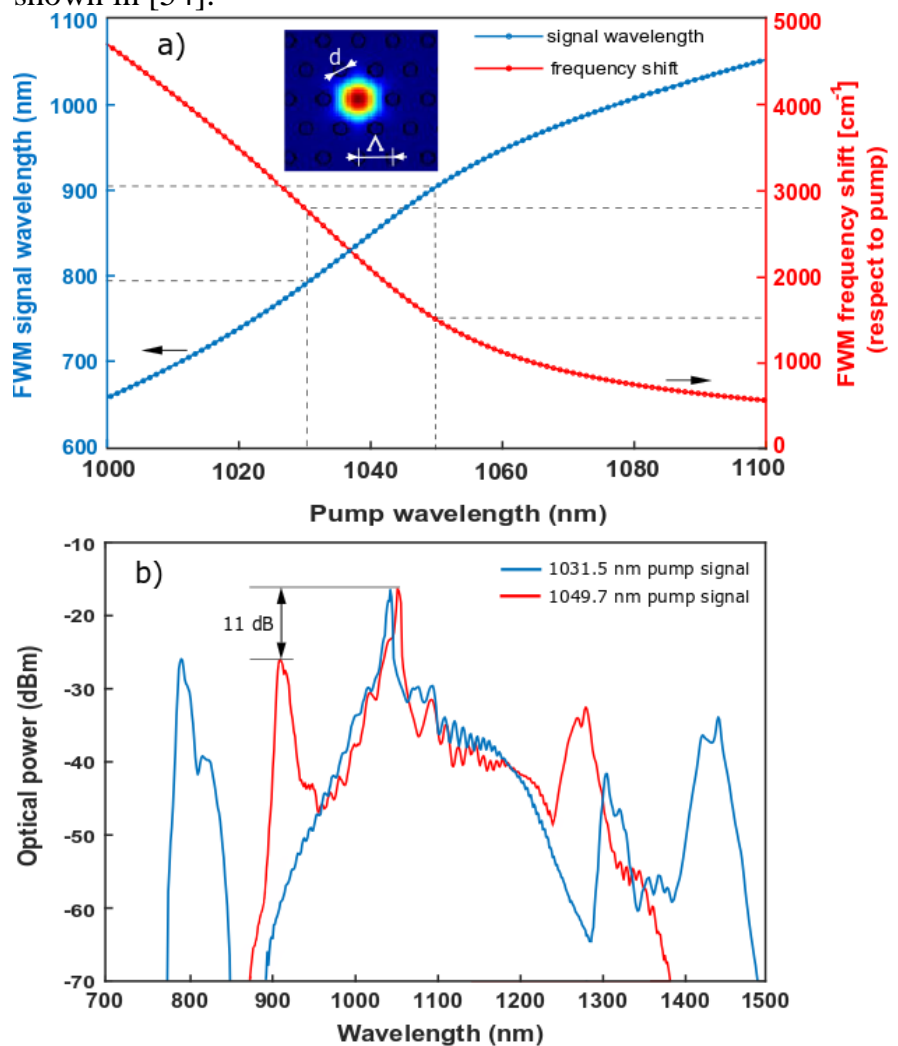

Fig.7. a) FWM phase-matching diagram of the anti-Stokes signal generated and FWM frequency shift respect to the pump in LMA-5-PM calculated for a peak power of $6 \mathrm{~kW}$ and b) Experimental results of the OPG based on DFWM at both wavelengths in the PCF LMA-5-PM 


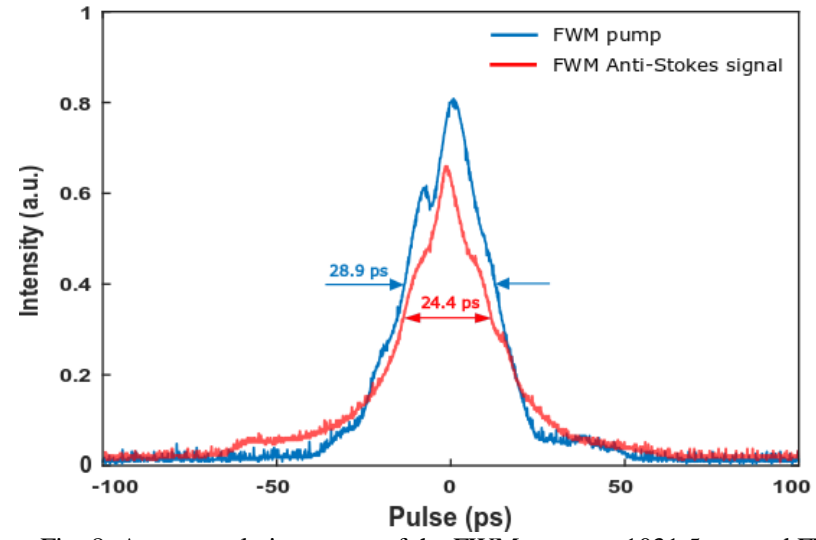

Fig. 8. Auto-correlation traces of the FWM pump at $1031.5 \mathrm{~nm}$ and FWM anti-Stokes signal generated at $794 \mathrm{~nm}$.

In addition, to control a precise FWM signal generation, the master oscillator can be tuned at both wavelengths through temperature variation of the FBGs, achieved with the aforementioned Peltier device. The tunable range of the $1031.5 \mathrm{~nm}$ oscillator emission is shown in Fig. 8). As it can be seen, temperature control allows for wavelength selection in a small range of $0.6 \mathrm{~nm}$ with a variation of $10 \mathrm{pm} /{ }^{\circ} \mathrm{C}$, which provides a precise adjustment to perfectly match FMW signal generation. In fact, this adjustment is crucial in order to obtain the maximum signal to noise ratio in CARS microscopy images, ensuring an overall higher quality contrast imaging.

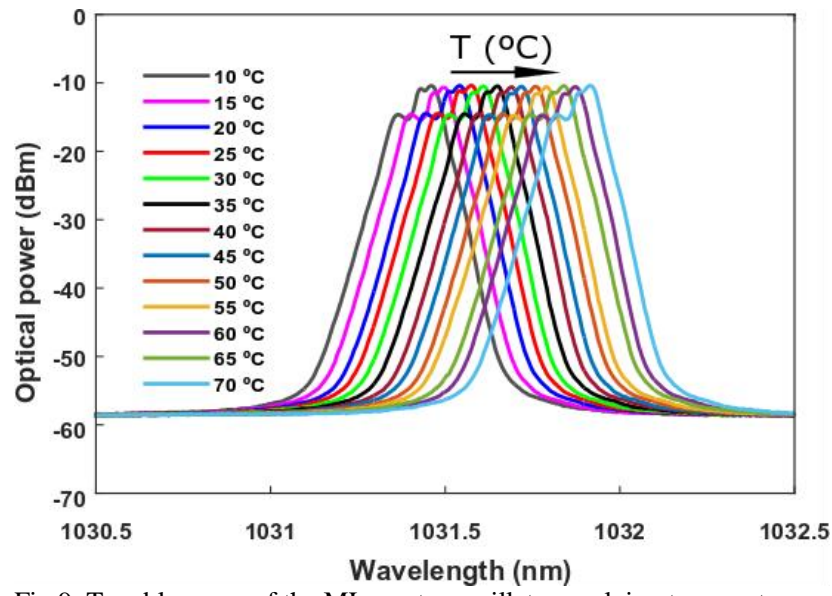

Fig 9. Tunable range of the ML master oscillator applying temperature on the FBG through a Peltiere device.

\section{CONCLUSIONS}

A self-started switchable dual-wavelength and low-cost ML fiber laser for optical parametric generation in PCF using only commercially available PM components has been presented. The proposed laser source has been demonstrated by combining conventional YDFA with a SESAM to generate mode-locking, and a pair of FBGs in parallel connection for wavelength selection. The herein described laser structure relies on a passively linear cavity oscillator with a low power threshold lees than $33 \mathrm{~mW}$, as well as a repetition rate of 3.1 $\mathrm{MHz}$ and pulse duration of $30.9 \mathrm{ps}$ and 31.6 at $1031.5 \mathrm{~nm}$ and $1049.7 \mathrm{~nm}$ respectively, which is directly amplified in a twostage YDFA reaching a peak power of more than $6 \mathrm{~kW}$ and a high slope-efficiency of $43 \%$. The described characteristics of this all-fiber free-alignment system allows for OPG in PCF LMA-5-PM, generating a frequency difference between the FWM anti-Stokes signal generated and the pump, which matches CARS resonances of $2850 \mathrm{~cm}^{-1}$ and $1475 \mathrm{~cm}^{-1}$ for each pump wavelength emission. The generated anti-Stokes signals have a $3-\mathrm{dB}$ spectral width of $4.1 \mathrm{~nm}$ and $4.6 \mathrm{~nm}$ at $1031.5 \mathrm{~nm}$ and $1049.7 \mathrm{~nm}$ of pump wavelength respectively. The pulse duration of the generated signals $(24.4 \mathrm{ps}$ at $794 \mathrm{~nm}$ and $23.1 \mathrm{ps}$ at $903 \mathrm{~nm}$ ) ensures a good overlapping for the CARS process. The temperature variation of the FBGs through a Peltier device grants accurate wavelength selection of the laser source in a $0.6 \mathrm{~nm}$ tuning range, matching the required CARS resonance with high precision to obtain the best contrast imaging. This simple, compact and reliable structure is appealing for nonlinear applications, particularly in-fiber FWM generation applied to CARS microscopy.

\section{REFERENCES}

[1] W. Kai-Hua, J. Pei-Pei, W. Bo, C. Tao, and S. Yong-Hang, "Fiber laser pumped burst-mode operated picosecond mid-infrared laser," Chinese Physics B, vol. 24, p. 024217, 2015.

[2] G. Whitenett, G. Stewart, H. Yu, and B. Culshaw, "Investigation of a tuneable mode-locked fiber laser for application to multipoint gas spectroscopy," Journal of Lightwave Technology, vol. 22, pp. 813819, 2004.

[3] U. Keller, "Recent developments in compact ultrafast lasers," Nature, vol. 424, p. 831, 2003.

[4] M. Azimipour and R. Pashaie, "Nonlinear optical signal processing on multiwavelength sensitive materials," Optics letters, vol. 38, pp. 4324-4326, 2013.

[5] J. Veselka and S. Korotky, "A multiwavelength source having precise channel spacing for WDM systems," IEEE Photonics Technology Letters, vol. 10, pp. 958-960, 1998.

[6] S. Rota-Rodrigo, I. Ibañez, and M. López-Amo, "Multiwavelength fiber laser in single-longitudinal mode operation using a photonic crystal fiber Sagnac interferometer," Applied Physics B, vol. 110, pp. 303-308, 2013.

[7] Y.-G. Han, T. Tran, S.-H. Kim, and S. B. Lee, "Multiwavelength Raman-fiber-laser-based long-distance remote sensor for simultaneous measurement of strain and temperature," Optics letters, vol. 30, pp. 1282-1284, 2005.

[8] M. Baumgartl, M. Chemnitz, C. Jauregui, T. Meyer, B. Dietzek, J. Popp, et al., "All-fiber laser source for CARS microscopy based on fiber optical parametric frequency conversion," Optics express, vol. 20, pp. 4484-4493, 2012.

[9] O. Kimmelma, S. C. Kumar, A. Esteban-Martin, and M. EbrahimZadeh, "Multi-gigahertz picosecond optical parametric oscillator pumped by $80-\mathrm{MHz}$ Yb-fiber laser," Optics letters, vol. 38, pp. 4550-4553, 2013.

[10] O. Kokabee, A. Esteban-Martin, and M. Ebrahim-Zadeh, "Efficient, high-power, ytterbium-fiber-laser-pumped picosecond optical parametric oscillator," Optics letters, vol. 35, pp. 3210$3212,2010$.

[11] T. Lamour, L. Kornaszewski, J. Sun, and D. Reid, "Yb: fiber-laserpumped high-energy picosecond optical parametric oscillator," Optics express, vol. 17, pp. 14229-14234, 2009.

[12] K. Wei, T. Chen, P. Jiang, D. Yang, B. Wu, and Y. Shen, "Fiber laser pumped high power mid-infrared laser with picosecond pulse bunch output," Optics express, vol. 21, pp. 25364-25372, 2013.

[13] L. Zhi-Chao, X. Wen-Cheng, S. Chuang-Xing, L. Ai-Ping, and C. Wei-Cheng, "Pulse-train nonuniformity in an all-fiber ring laser passively mode-locked by nonlinear polarization rotation," Chinese Physics B, vol. 18, p. 2328, 2009.

[14] L. Kong, X. Xiao, and C. Yang, "Low-repetition-rate all-fiber allnormal-dispersion Yb-doped mode-locked fiber laser," Laser Physics Letters, vol. 7, pp. 359-362, 2010. 
[15] K. Tamura, E. Ippen, H. Haus, and L. Nelson, "77-fs pulse generation from a stretched-pulse mode-locked all-fiber ring laser," Optics letters, vol. 18, pp. 1080-1082, 1993.

[16] J. Travers, E. Obraztsova, A. Lobach, A. Chernov, E. Kelleher, S. Popov, et al., "Mode-Locking Fibre Lasers with the E Transition of Carbon Nanotubes," in The European Conference on Lasers and Electro-Optics, 2009, p. CJ10_1.

[17] J.-C. Chiu, Y.-F. Lan, J.-J. Kang, C.-M. Chang, Z.-S. Haung, C.-Y. Yeh, et al., "Passively mode-locked lasers using saturable absorber incorporating dispersed single-wall carbon nanotubes," in Electronic Components and Technology Conference, 2009. ECTC 2009. 59th, 2009, pp. 827-830.

[18] W. B. Cho, H. W. Lee, S. Y. Choi, J. W. Kim, D.-I. Yeom, F. Rotermund, et al., "Monolayer graphene saturable absorber for bulk laser mode-locking," in Conference on Lasers and ElectroOptics, 2010, p. JThE86.

[19] S. Zhipei, T. Hasan, D. Popa, F. Torrisi, F. Wang, F. Bonaccorso, et al., "Ultrafast fiber laser mode-locked by graphene based saturable absorber," in Conference on Lasers and Electro-Optics, 2010, p. CTuR1.

[20] U. Keller, K. J. Weingarten, F. X. Kartner, D. Kopf, B. Braun, I. D. Jung, et al., "Semiconductor saturable absorber mirrors (SESAM's) for femtosecond to nanosecond pulse generation in solid-state lasers," IEEE Journal of selected topics in QUANTUM ELECTRONICS, vol. 2, pp. 435-453, 1996.

[21] A. Agnesi, L. Carra, C. Marco, R. Piccoli, and G. Reali, "FourierLimited 19-ps Yb-Fiber Seeder Stabilized by Spectral Filtering and Tunable Between 1015 and 1085 nm," IEEE Photonics Technology Letters, vol. 24, p. 927, 2012.

[22] U. Keller, "Ultrafast solid-state laser oscillators: a success story for the last 20 years with no end in sight," Applied Physics B, vol. 100, pp. 15-28, 2010.

[23] O. Okhotnikov, A. Grudinin, and M. Pessa, "Ultra-fast fibre laser systems based on SESAM technology: new horizons and applications," New journal of physics, vol. 6, p. 177, 2004.

[24] K. Viskontas and N. Rusteika, "All-fiber wavelength-tunable picosecond nonlinear reflectivity measurement setup for characterization of semiconductor saturable absorber mirrors," Optical Fiber Technology, vol. 31, pp. 74-82, 2016.

[25] J. Limpert, F. Roser, T. Schreiber, and A. Tunnermann, "Highpower ultrafast fiber laser systems," IEEE Journal of selected topics in Quantum Electronics, vol. 12, pp. 233-244, 2006.

[26] R. Paschotta, J. Nilsson, A. C. Tropper, and D. C. Hanna, "Ytterbium-doped fiber amplifiers," IEEE Journal of quantum electronics, vol. 33, pp. 1049-1056, 1997.

[27] P. Peterka, J. Maria, B. Dussardier, R. Slavik, P. Honzatko, and V. Kubeček, "Long-period fiber grating as wavelength selective element in double-clad Yb-doped fiber-ring lasers," Laser Physics Letters, vol. 6, pp. 732-736, 2009.

[28] S. Harun, M. Moghaddam, K. Dimyati, and H. Ahmad, "The performance of double-clad ytterbium-doped fiber laser with different pumping wavelengths," Laser Physics Letters, vol. 6, p. 458, 2009.

[29] S. Kobtsev, S. Kukarin, and S. Smirnov, "All-fiber high-energy supercontinuum pulse generator," Laser Physics, vol. 20, pp. 375$378,2010$.

[30] P. Jiang, D. Yang, Y. Wang, T. Chen, B. Wu, and Y. Shen, "Allfiberized MOPA structured single-mode pulse $\mathrm{Yb}$ fiber laser with a linearly polarized output power of $30 \mathrm{~W}$," Laser Physics Letters, vol. 6, pp. 384-387, 2009.

[31] G. Jang and T. Yoon, "Environmentally-stable all-normaldispersion picosecond $\mathrm{Yb}$-doped fiber laser with an achromatic quarter-wave-plate," Laser Physics, vol. 20, pp. 1463-1468, 2010.

[32] M. E. Fermann and I. Hartl, "Ultrafast fiber laser technology," IEEE Journal of Selected Topics in Quantum Electronics, vol. 15, pp. 191-206, 2009.

[33] L. Wang, X. Liu, and Y. Gong, "Giant-chirp oscillator for ultralarge net-normal-dispersion fiber lasers," Laser Physics Letters, vol. 7, p. 63, 2009.

[34] M. Salhi, A. Haboucha, H. Leblond, and F. Sanchez, "Theoretical study of figure-eight all-fiber laser," Physical Review A, vol. 77, p. 033828, 2008.

[35] M. Ahmed, N. Ali, Z. Salleh, A. Rahman, S. Harun, M. Manaf, et al., "All fiber mode-locked Erbium-doped fiber laser using singlewalled carbon nanotubes embedded into polyvinyl alcohol film as saturable absorber," Optics \& Laser Technology, vol. 62, pp. 4043, 2014.

[36] A. Agnesi, L. Carrá, F. Pirzio, R. Piccoli, and G. Reali, "Low repetition rate, hybrid fiber/solid-state, $1064 \mathrm{~nm}$ picosecond master oscillator power amplifier laser system," JOSA B, vol. 30, pp. 2960-2965, 2013.

[37] X. Liu, "Pulse evolution without wave breaking in a strongly dissipative-dispersive laser system," Physical Review A, vol. 81, p. $053819,2010$.

[38] X. Liu, "Interaction and motion of solitons in passively-modelocked fiber lasers," Physical Review A, vol. 84, p. 053828, 2011.

[39] Z.-C. Luo, A.-P. Luo, and W.-C. Xu, "Tunable and switchable multiwavelength passively mode-locked fiber laser based on SESAM and inline birefringence comb filter," IEEE Photonics Journal, vol. 3, pp. 64-70, 2011.

[40] J. Lægsgaard, "Control of fibre laser mode-locking by narrowband Bragg gratings," Journal of Physics B: Atomic, Molecular and Optical Physics, vol. 41, p. 095401, 2008.

[41] I. A. Litago, D. Leandro, M. Á. Quintela, R. A. Pérez-Herrera, M. López-Amo, and J. M. López-Higuera, "Tunable SESAM-based mode-locked soliton fiber laser in linear cavity by axial-strain applied to an FBG," Journal of Lightwave Technology, vol. 35, pp. 5003-5009, 2017.

[42] G. A. Cranch, G. M. Flockhart, and C. K. Kirkendall, "Efficient fiber Bragg grating and fiber Fabry-Perot sensor multiplexing scheme using a broadband pulsed mode-locked laser," Journal of lightwave technology, vol. 23, p. 3798, 2005.

[43] J. Canning, "Fibre gratings and devices for sensors and lasers," Laser \& Photonics Reviews, vol. 2, pp. 275-289, 2008.

[44] X. Liu and D. Mao, "Compact all-fiber high-energy fiber laser with sub-300-fs duration," Optics express, vol. 18, pp. 8847-8852, 2010.

[45] M. Baumgartl, J. Abreu-Afonso, A. Díez, M. Rothhardt, J. Limpert, and A. Tünnermann, "Environmentally stable picosecond $\mathrm{Yb}$ fiber laser with low repetition rate," Applied Physics B, vol. 111, pp. 39-43, 2013.

[46] T. Gottschall, M. Baumgartl, A. Sagnier, J. Rothhardt, C. Jauregui, J. Limpert, et al., "Fiber-based source for multiplex-CARS microscopy based on degenerate four-wave mixing," Optics Express, vol. 20, pp. 12004-12013, 2012.

[47] S. Lefrancois, D. Fu, G. R. Holtom, L. Kong, W. J. Wadsworth, P. Schneider, et al., "Fiber four-wave mixing source for coherent anti-Stokes Raman scattering microscopy," Optics letters, vol. 37, pp. 1652-1654, 2012.

[48] L. Talaverano, S. Abad, S. Jarabo, and M. Lopez-Amo, "Multiwavelength fiber laser sources with Bragg-grating sensor multiplexing capability," Journal of lightwave technology, vol. 19, pp. 553-558, 2001.

[49] E. Achaerandio, S. Jarabo, S. Abad, and M. López-Amo, "New WDM amplified network for optical sensor multiplexing," IEEE Photonics Technology Letters, vol. 11, pp. 1644-1646, 1999.

[50] X.-D. Wu, C. Schmidt-Hattenberger, K. Krüger, and J. Chen, "Temperature-controlled fiber Bragg grating dynamic strain detection system," Sensors and Actuators A: Physical, vol. 119, pp. 68-74, 2005.

[51] Y. He and F. Shi, "Finite-difference imaginary-distance beam propagation method for modeling of the fundamental mode of photonic crystal fibers," Optics communications, vol. 225, pp. 151156,2003

[52] K. Saitoh and M. Koshiba, "Empirical relations for simple design of photonic crystal fibers," Optics express, vol. 13, pp. 267-274, 2005.

[53] E. A. Zlobina, S. I. Kablukov, and S. A. Babin, "Phase matching for parametric generation in polarization maintaining photonic crystal fiber pumped by tunable Yb-doped fiber laser," JOSA B, vol. 29, pp. 1959-1967, 2012.

[54] T. Gottschall, T. Meyer, M. Baumgartl, C. Jauregui, M. Schmitt, J. Popp, et al., "Fiber-based light sources for biomedical applications of coherent anti-Stokes Raman scattering microscopy," Laser \& Photonics Reviews, vol. 9, pp. 435-451, 2015. 